\title{
Investigation into the Optimal Wind Turbine Layout Patterns for a Wind Farm in Walvis Bay, Namibia
}

\author{
Tom Wanjekeche \\ Department of Electrical Engineering, University of Namibia, Ongwediva, Namibia
}

Email address:

wanjekeche $a$ yahoo.com

\section{To cite this article:}

Tom Wanjekeche. Investigation into the Optimal Wind turbine layout patterns for a Wind farm in Walvis Bay, Namibia. American Journal of Electrical Power and Energy Systems. Vol. 7, No. 3, 2018, pp. 33-41. doi: 10.11648/j.epes.20180703.12

Received: June 13, 2018; Accepted: August 3, 2018; Published: August 30, 2018

\begin{abstract}
Due to the unpredictable nature of wind speed and direction, there is a need to optimize the wind turbines placement to extract maximum available wind power at a low cost. Through optimization, best positions of the wind turbines that lead to maximum output are determined. This paper presents an into an optimal Wind Turbine (WT) layout pattern for three Wind Farm (WF) configurations (aligned, Genetic Algorithm (GA) and Particle Swarm Optimization (PSO). A Hypothetical WF (2km X $2 \mathrm{~km}$ ) is analyzed based on 2016 Wind data. Result shows that the total power generated from the customized is $863.098 \mathrm{~kW}$, from Genetic Algorithm (GA) layout, the total power generated is $1296.286 \mathrm{~kW}$ while from Particle Swarm Optimization (PSO) the total power generated is $1300.668 \mathrm{~kW}$. In comparison to the customized layout, optimization algorithms layouts resulted in a good improvement of the total power generated, GA improved the total power generated by $50.2 \%$ while PSO improved the total power generated by 50.7\%. Optimization Algorithms layout proved to be efficient as compared to the customized layout because they have fewer power losses. GA and PSO layout have losses of 13.5\% and 13.3\% respectively, while the customized layout resulted in the most losses which are at $43 \%$. The results from GA and PSO slightly differ, with a small difference in power of 4.4 $\mathrm{kW}$.
\end{abstract}

Keywords: Wind Turbine, Optimization, Genetic Algorithm, Particle Swarm Optimization, Optimal Arrangement

\section{Introduction}

Namibia has set itself an ambitious target to have $70 \%$ of energy needs met by renewable energy sources by 2020 [1] With abundant resources such as wind along the coastline of the country, Namibia's energy deficit can be solved by harnessing such free resources by means of wind farms. Due to the unpredictable nature of wind speed and direction, there is a need to optimize the wind turbines placement to extract maximum available wind power at a low cost. Through optimization, best positions of the wind turbines that lead to maximum output are determined [2]. Walvis Bay is one of the well-established towns in Namibia, this town is located on the coast of the Atlantic Ocean in Erongo Region, Namibial

\subsection{Wind Farm Overview}

Nowadays, energy from wind is produced on large scale, a large number of turbines are now usually installed together at a site with good wind speed, and the installation is referred to as a wind farm. The advantage of clustering turbines together in a wind farm is because it leads to reduced site development costs, it simplifies connections to the grid and it gives an easier access for personnel to operate and do maintenance on the wind farm [3]

\subsubsection{Weibull Distribution Function Parameters for Wind Energy Analysis}

Several tools are utilised for wind speed data analysis. From these tools, two parameters of the Weibull distribution function are mostly used for analysing measured wind speed data from an area over a given period of time. The Weibull distribution function is preferred as it gives a better fitting and high accuracy for monthly probability density distributions of measured wind speed than any other distribution function [4] The Weibull distribution function is given by:

$$
f(v)=\left(\frac{k}{c}\right)\left(\frac{v}{c}\right)^{k-1} \exp \left[-\left(\frac{v}{c}\right)^{k}\right]
$$

The shape parameter, $k$ estimates how good the wind blows 
while the scale parameter, $c$ estimates how hard wind blows [3]

\subsubsection{Turbine Placement}

Performance of large wind farms is entirely determined by the positioning of their turbines [5]. Optimum arrangement of wind turbines in a wind farm is done by optimization algorithms that choose the best turbine positions. Optimal placement for wind turbines depends on the choice of the number of turbines and the turbine type, putting into consideration the given requirements and restrictions. To determine the optimum relation between turbines, the investment cost and total power of the wind farm, wind turbines numbers and type are used as the optimization constraints [6].

The wind turbine arrangement optimization problem is solved using mathematical models. During the optimization process, the mathematical model uses one of the two basic approaches in determining the solution; these approaches are the discrete model and the continuous model approach [7].

\subsection{Wind Farm Optimization}

The first step to begin with when using optimization algorithms to solve problems is to define the objective function or the standard requirement that the wind farm must meet in order for the solution to be considered optimum. The most commonly used objective functions are, the energy production, cost of energy, profit maximization or a combination of the any of the stated approach [8]

Jun Wang and colleagues [9] used the energy production approach to develop the objective function. The turbine placement problem was solved by setting up an allowed minimum distance $d_{\min }$ between turbines and the optimal turbine arrangement problem was to maximize the power produced by the wind farm which is given by:

$$
\max P(Z)
$$

Subjected to the following constraints on turbine positions

$$
\begin{gathered}
\left\{\begin{array}{c}
g_{k}(Z)=\left(x_{m-} x_{n}\right)^{2}+\left(y_{m-} y_{n}\right)^{2}-d_{\text {min }}^{2} \geq 0 \\
0 \leq x_{m} \leq X_{\max }, 0 \leq y_{m} \leq Y_{\max }
\end{array}\right. \\
(k \in\{1,2, \ldots ., C\}, \forall m \neq n, m, n \in\{1,2, \ldots, N\})
\end{gathered}
$$

Pierre-Elouan Rethore and colleagues [10] used the financial balance function to model the profit of an offshore wind farm. In this work, the objective function was aimed to the optimization of the wind farm profit by balancing the energy production income, electrical grid costs, foundation cost and the cost of wake turbulence induced fatigue degradation of different wind turbine components. The financial balance function is given by:

$$
F B=W P_{n}-C\left(1+\left(\frac{r_{c}-r_{i}}{n_{L}}\right)\right)^{X_{n L}}
$$

The net value of the power production is defined as:

$$
\mathrm{WPn}=\mathrm{WP}-\mathrm{CD}-\mathrm{CM}
$$

Where $W P$ is the value of the wind farm power production over the wind farm lifetime, $C D$ is the accumulated cost of components degradation

J. Serrano Gonzalez and colleagues [11], in their work, they based the objective function that they used to the Net Present Value (NPV) of the wind farm. The objective of solving the optimization problem was to optimize the wind farm profits given an investment cost of the wind farm. For the NPV to be determined, the initial capital investment and net cash flow throughout the wind farm life cycle were calculated. NPV is maximized when the investment cost is minimized and when the net cash flow is maximized.

\section{Materials and Methodology}

A quantitative study was used whereby wind data for Walvis Bay were collected from The Namibian Metrological Services (NMS). This was used to determine the constant wind speed. The wind farm design was carried out by considering the wind farm area, the number of turbines to be placed in the wind farm and the wind direction. A customized wind farm with turbines that are arranged with equal spacing between them was done. The optimization of the wind turbine placement in a wind farm was achieved by using an objective function that denotes the cost per unit power produced by the wind farm. Two optimization algorithms, Genetic algorithm (GA) and Particle Swarm Optimization Algorithm (PSO) were used for optimally placing wind turbines in a wind farm. The Jansen's wake effect model was used for modeling wind turbine wakes in the wind farm.

\subsection{Determination of the Wind Speed Used}

2016 wind speed data for Walvis Bay were obtained as given in table 1 . The shape (k) and scale (c) parameters from the Weibull distribution function were used to determine the constant wind velocity used [4]. The Weibull distribution function is given (1), whereby $\mathrm{k}$ and $\mathrm{c}$ are given by:

$$
\begin{gathered}
k=\left(\frac{\sigma}{V_{m}}\right)^{-1.086} \\
c=\frac{V_{m}}{\Gamma\left(1+\frac{1}{k}\right)}
\end{gathered}
$$

Table 1. 2016 Monthly Wind Velocity for Walvis Bay.

\begin{tabular}{ll}
\hline Month & Wind Velocity $(\mathbf{m} / \mathbf{s})$ \\
\hline January & 3.2 \\
February & 3.2 \\
March & 2.5 \\
April & 2.4 \\
May & 3.3 \\
June & 3.2 \\
July & 3.5 \\
August & 2.8 \\
September & 3.1 \\
October & 3 \\
November & 3.5 \\
December & 3.6 \\
\hline
\end{tabular}


By which $\sigma$ is the variance, $V_{m}$ is the mean wind velocity.

The values of $\mathrm{c}$ and $\mathrm{k}$ lead to the determination of the constant wind velocity that was used for this study by using:

$$
V=c\left(\frac{k+2}{k}\right)^{\frac{1}{k}}
$$

Using the Weibull distribution and Table 1, the wind parameters for Walvis bay are computed as:

Mean wind speed was determined by:

$$
\begin{array}{r}
V_{m}=\frac{1}{N}\left(\sum_{i=1}^{N} V_{i}\right) \\
V_{m}=3.1 \mathrm{~m} / \mathrm{s}
\end{array}
$$

The variance was determined by:

$$
\begin{gathered}
\sigma=\left[\frac{1}{N-1} \sum_{i=1}^{N} V_{i}-V_{m}\right] \\
\sigma=0.3639
\end{gathered}
$$

From (6)

$$
k=10.24
$$

From (7)

$$
\begin{gathered}
c=\frac{3.1}{0.934} \\
c=3.25 \mathrm{~m} / \mathrm{s}
\end{gathered}
$$

From (8)

$$
\begin{gathered}
V=3.25\left(\frac{10.24+2}{10.24}\right)^{\frac{1}{10.24}} \\
V=4.14 \mathrm{~m} / \mathrm{s}
\end{gathered}
$$

\subsection{Wind Farm Design}

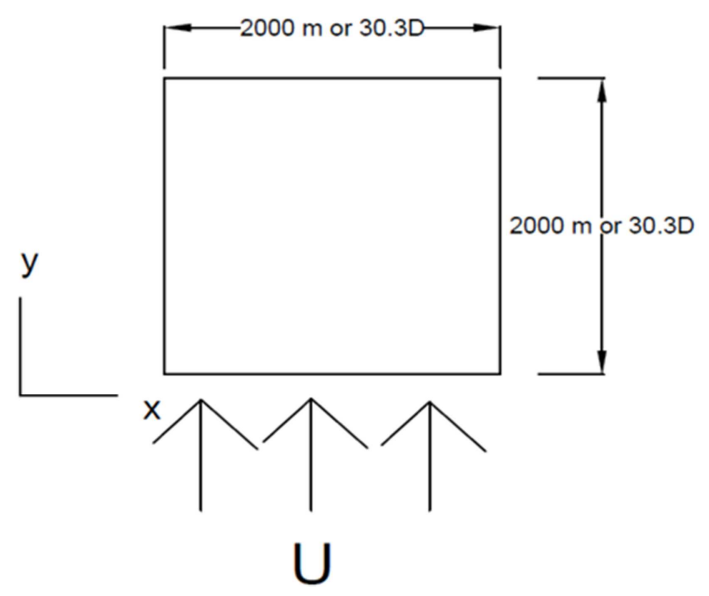

Figure 1. Wind farm area.

The wind farm design was made under some assumptions. The area of the wind farm was assumed, the number of turbines to be placed in the wind farm were assumed and the wind direction was also assumed to be constant. The wind farm area determines the space available for placing turbines, for this study the wind farm area was assumed to be of a $2 \mathrm{~km}$ by $2 \mathrm{~km}$ square. The number of turbines were set to be 30 and the wind direction was set to be in the $\mathrm{y}-$ axis direction. The wind farm area and the wind direction are shown in figure 1.

\subsection{Wind Farm Design}

The wind farm design was made under some assumptions. The area of the wind farm was assumed, the number of turbines to be placed in the wind farm were assumed and the wind direction was also assumed to be constant. The wind farm area determines the space available for placing turbines, for this study the wind farm area was assumed to be of a $2 \mathrm{~km}$ by $2 \mathrm{~km}$ square. The number of turbines were set to be 30 and the wind direction was set to be in the $\mathrm{y}-$ axis direction. The wind farm area and the wind direction are shown in figure 1.

\subsection{Customized Wind Farm Turbine Spacing}

The customized wind farm is made up of turbines that are equally spaced between the columns and between the rows. For the 30 turbines to be arranged in a wind farm of an area of $2 \mathrm{~km} \times 2 \mathrm{~km}$ with equal distances between them, the technique for spacing turbine using the separation distance of 5-10 D was employed (whereby D is the turbine rotor diameter in meters [3]. For 30 turbines to be arranged with equal spacing between them, a topology of 6 turbines in each row and 5 turbines in each column was used.

Since each length of the wind farm is $2000 \mathrm{~m}$, turbines on the side of 6 require a separation distance of $333 \mathrm{~m}$ (i.e. 5.05D), while Turbines on the side of 5 require a separation distance of $400 \mathrm{~m}$ (i.e. 6.06D). The $333 \mathrm{~m}$ on the $\mathrm{x}$ - axis direction is regarded as crosswind direction while the $400 \mathrm{~m}$ on the $y$ - axis direction is regarded as downwind direction.

The turbine used for this study was determined by using the minimal separation distance for arranging turbines with equal spacing of $333 \mathrm{~m}$ or $5.05 \mathrm{D}$. Based on the chosen separation distance, the applicable wind turbine requires a rotor diameter of $66 \mathrm{~m}$. A Vestas turbine was selected and its parameters are listed in table 2 .

Table 2. Turbine Parameters.

\begin{tabular}{ll}
\hline Turbine Parameter & Value \\
\hline Hub height & $73 \mathrm{~m}$ \\
Rotor radius & $33 \mathrm{~m}$ \\
Thrust coefficient & 0.888 \\
Cut in wind speed & $3 \mathrm{~m} / \mathrm{s}$ \\
Cut out wind speed & $34 \mathrm{~m} / \mathrm{s}$ \\
Rated wind speed & $4 \mathrm{~m} / \mathrm{s}$ \\
Rated Power & $50 \mathrm{~kW}$ \\
\hline
\end{tabular}

\subsection{Optimization of the Wind Turbine Layout}

\subsubsection{Optimization Algorithms}

Two optimization algorithms, Genetic algorithm (GA) and Particle Swarm Optimization Algorithm (PSO) were used for optimally placing wind turbines in a wind farm.

The use of GA in the optimization of turbines arrangement in a wind farm was first proposed by Mosetti [5]. The purpose of this study was to prove the feasibility of using Genetic Algorithm in the optimal placement of wind turbines in a wind 
farm. The optimal arrangement of wind turbines in a given site was done in order to maximize the power yield from the wind farm at minimum wind farm investment costs. Generic algorithm was used to optimize the placement. Mosetti's results were optimal as the wind farm developed was of high efficiency.

Mosetti's study was improved by S. A. Grady Genetic Algorithm was employed to optimally arrange wind turbines in a wind farm to achieve maximum power production while reducing the number of turbines placed in the wind farm. The results of S. A. Grady showed an improvement of Mosetti's study, the improvement occurred because S. A. Grady work was allowed to run for many generations as compared to Mosetti's work [5, 13].

Rabia Shakoor, Mohammad Yusri Hassan, Abdur Raheem, Nadia Rasheed and Mohamad Na'im Mohd Nasir used Definite Point Selection (DPS) and Genetic Algorithm to optimally arrange wind turbines in a wind farm by minimizing the cost per unit power and minimizing the wake effects [14]. The cost per unit power was minimized by changing the dimensions of the wind farm while keeping the area of the wind farm constant by which the area is the same as the area used in $[5,13]$. By comparing the results in [14] to the results in [8] and [13] that the power output of the wind farm increased by using the same area with different dimensions when the total number of wind turbines in a wind farm were the same.

The first attempt to apply PSO on wind farms was done by Jun Wang and Xing Zhang [9]. In their work, they used PSO to optimally arrange wind turbines in wind farm in which their objective function was to maximize power produced while keeping a specified minimum distance between turbines.

Jun Wang and Xing Zhang later reviewed their work and they made some improvements. In the second attempt, they used PSO with Deferential Evolution to develop layouts of wind farms with turbines that are optimally arranged $[15,16]$. From the obtained results, it shows that Deferential Evolution gave more power as compared to PSO.

\subsubsection{Objective Function Formulation}

For an optimization problem to be solved by using optimization algorithms, the most important parameter to consider is the objective function. The objective function helps the optimization algorithm to select the best results for the problem. The objective function is either maximized or minimized according to the nature of the problem. Objective function is given by:

$$
\text { Objective Function }=\frac{\text { Cost }}{\text { Total Power }}
$$

Cost is the investment cost of the wind farm and it is modeled by the equation below:

$$
\text { Cost }=N\left(\frac{2}{3}+\frac{1}{3} e^{-0.00174 N^{2}}\right)
$$

The investment cost is modeled in such a way that only the number of turbines is considered. From the investment cost, it is assumed that the dimensionless cost/year of a single turbine is 1 and the maximum cost reduction for each turbine is $1 / 3$ if many turbines are installed in a wind farm [9]. For this study, the investment cost is fixed because the number of turbines is constant.

Total power is the power produced by the wind and it is computed as follows: Power produced by one wind turbine is given by:

$$
\text { Power }=\eta \frac{1}{2} \rho A u^{3}
$$

Assuming the efficiency of the wind turbine used is $30 \%$ and using the standard air density and pressure, the power produced by one turbine is given by:

$$
\begin{gathered}
\text { Power }=\frac{30}{100} \times \frac{1}{2} \times 1.225 \times \pi \times 33^{2} \times u^{3} \\
\text { Power }=0.63 u^{3} \mathrm{~kW}
\end{gathered}
$$

Total power produced by a wind farm is given by:

$$
\text { Total Power }=\sum_{i}^{N} 0.63 u_{i}^{3} k W
$$

In a wind farm, downwind turbines receive modified wind speed which is low as compared to wind speed approaching upstream turbines, with this, each downwind turbine receives a unique wind speed this hence resulting in a unique power. This is caused by the wake effect. The wind speed developed at downwind turbines is estimated by using different wake effect models, for this study the Jensen's wake effect was used for calculating the downstream turbines [12].

The execution of the code is illustrated by the flowchart in figure 2. The steps used in computational procedure of the objective function are as follows:

Step 1: Turbines coordinates are received from the optimization algorithm or received when they are manually entered.

Step 2: Initialization of the power sum to zero and also the number of turbines to zero.

Step 3: A turbine of which its power is to be calculated is selected.

Step 4: A scenario is selected. This is based on how the turbine that is being executed is affected by the wind. A turbine is either experiencing no wake, experiencing a single wake or experiencing multiple wakes. respectively while for scenario 1, the freestream wind speed is used

Step 5: After the wind speed is determined, power produced by a turbine is calculated by using equation (18).

Step 6: Power of a turbine is added to the summation of the total power.

Step 7: If the number of turbines executed is less than 30 , go to step 3, otherwise, go to step 8 .

Step 8: Calculate the objective function value base on equation (13)

The computation procedure illustrated in figure 2 is a subroutine to the computation procedure illustrated in figure 3 . For the process illustrated in figure 2 to occur, certain parameters are required to be set for the process illustrated in figure 3 . 


\subsubsection{Optimization Algorithm Initialization}

Genetic algorithm

1. Population size: In this optimization problem, the population size was set to 60 which is twice the number of turbines in the wind farm, this is because the number of turbines is located by using $(x, y)$ coordinates, in GA the population size is referred to as the number of variables.

2. Limits: The limits were set to specify upper bound and the lower bound, the limits specify the wind farm region so that turbines are not placed outside of the wind farm region. For this optimization problem, the limits were set to range from 0 to $2 \mathrm{~km}$ in both $\mathrm{x}$ and $\mathrm{y}$ directions.

3. Optimization criteria: This is the optimization stopping criteria, for GA, the optimization criterion was set to terminate whenever if the average change in the best objective function value over stall generations is less than or equal to the function tolerance.

\section{Particle Swarm Optimization}

1. Population size: In this optimization problem, the population size was set to 60 which is twice the number of turbines in the wind farm, this is because the number of turbines is located by using $(x, y)$ coordinates, in PSO the population size is referred to as the number of particles.

2. Limits: The limits were set to specify upper bound and the lower bound, the limits specify the wind farm region so that turbines are not placed outside of the wind farm region. For this optimization, problem the limits were set to range from 0 to $2 \mathrm{~km}$ in both $\mathrm{x}$ and $\mathrm{y}$ directions.

3. Optimization criteria: This is the optimization stopping criteria, for PSO, the optimization criterion was set to terminate whenever the relative change in the best objective function value over the last maximum stall iterations is less than the function tolerance.
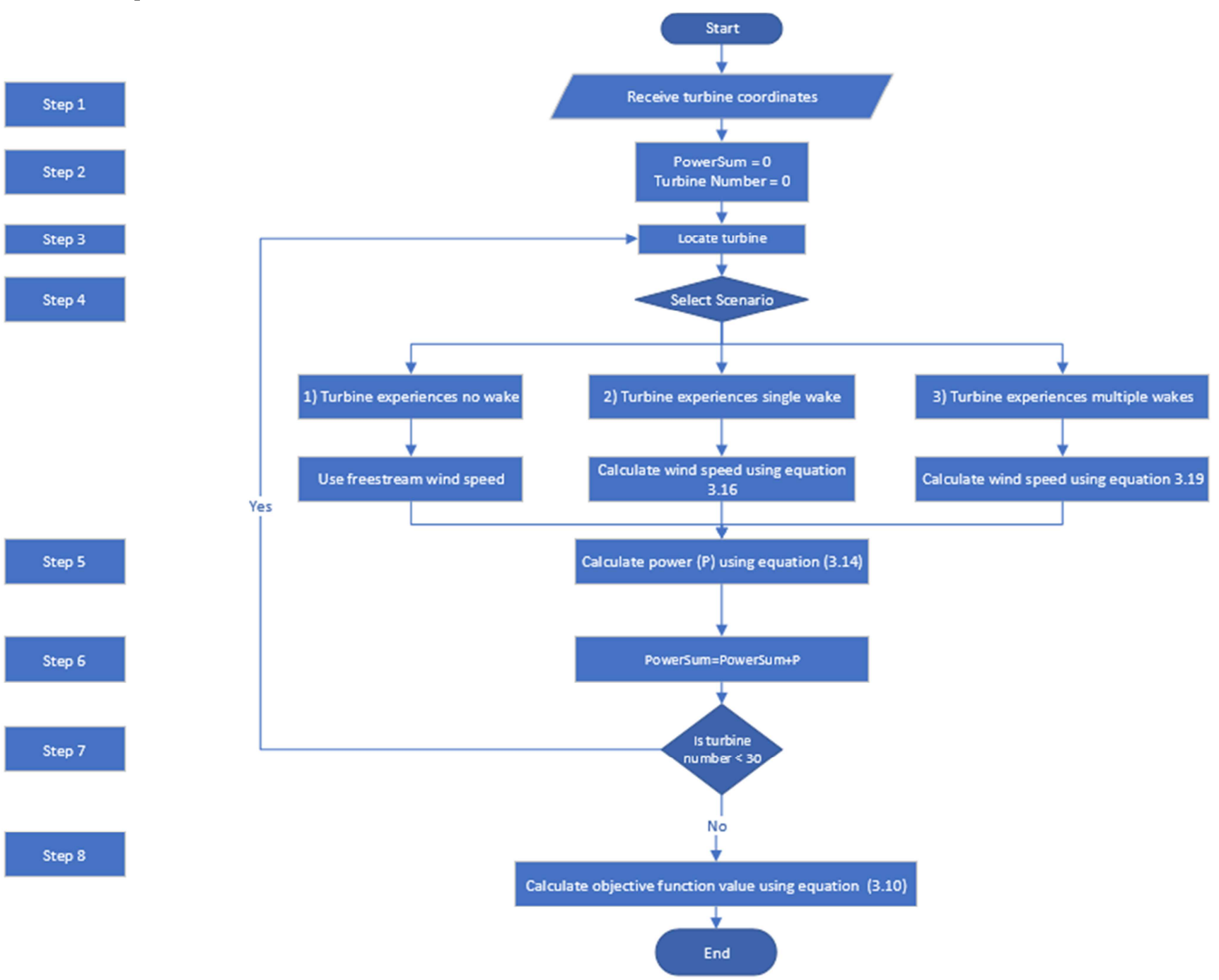

Figure 2. Objective Function Computation Procedure. 


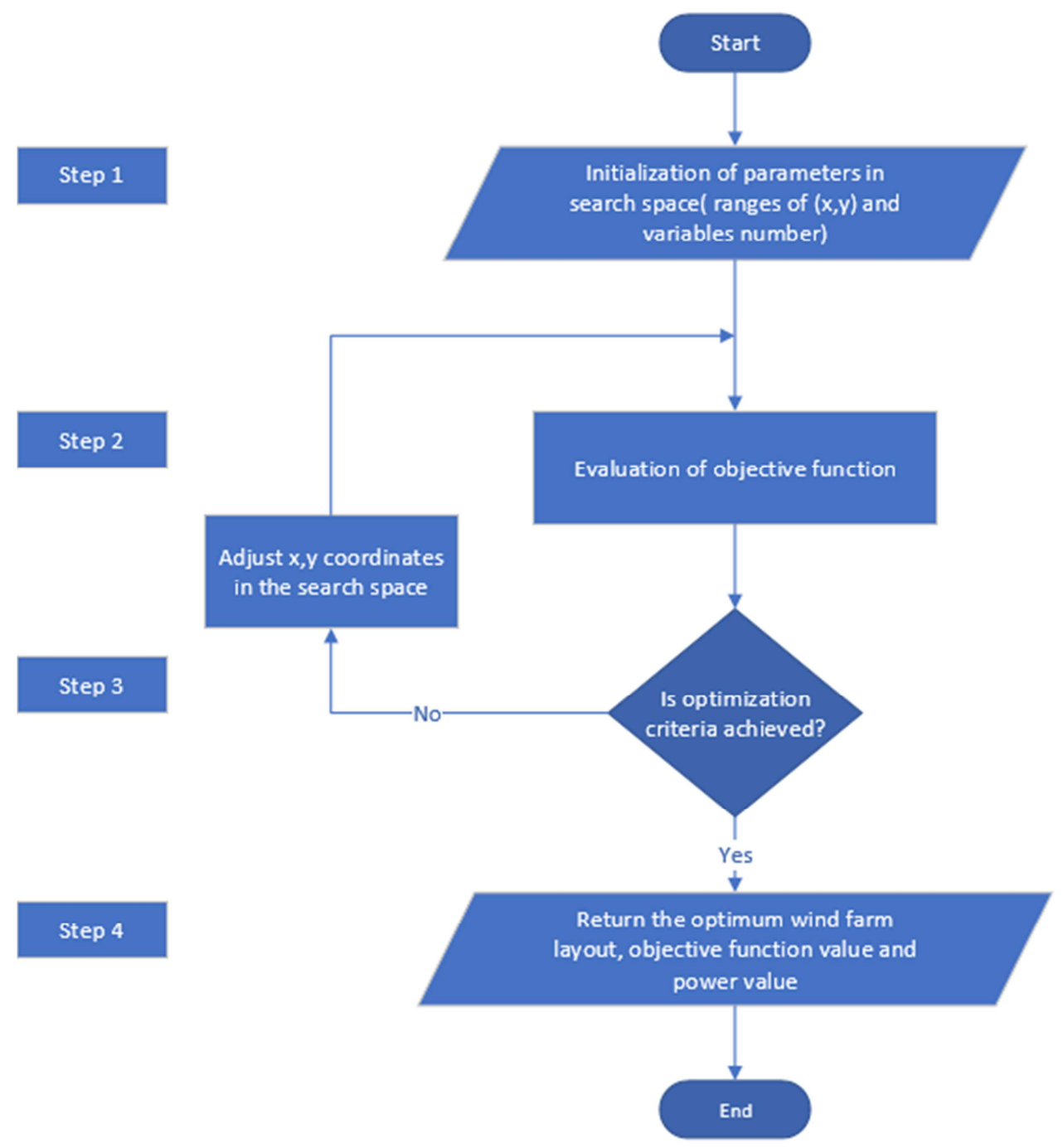

Figure 3. Optimization Algorithms Execution Procedure.

Figure 3 shows the optimization procedure and its execution is as given:

Step 1: In the search space, the ranges of $x$ and of $y$ are set together with the number of variables which represent the number of turbines.

Step 2: The objective function is evaluated based on the flowchart in figure 2

Step 3: The optimization criteria is achieved if: (for GA: the average change in the best objective function value over stall generations is less than or equal to the function tolerance and for PSO: the relative change in the best objective function value over the last maximum stall iterations is less than the function tolerance), go to step 4. Otherwise, the $x$ and $y$ coordinates are adjusted in the search space.

Step 4: Return the wind farm layout with turbines that are optimally arranged, the objective function value, and power value.

\section{Results and Discussion}

The results obtained from the customized wind farm were compared to the results obtained by using the optimization algorithms. Wind farm layouts were presented in figures showing the arrangement of wind turbines with their corresponding generated powers. The discussion of the arrangement for each wind farm layout was done to interpret the study findings.

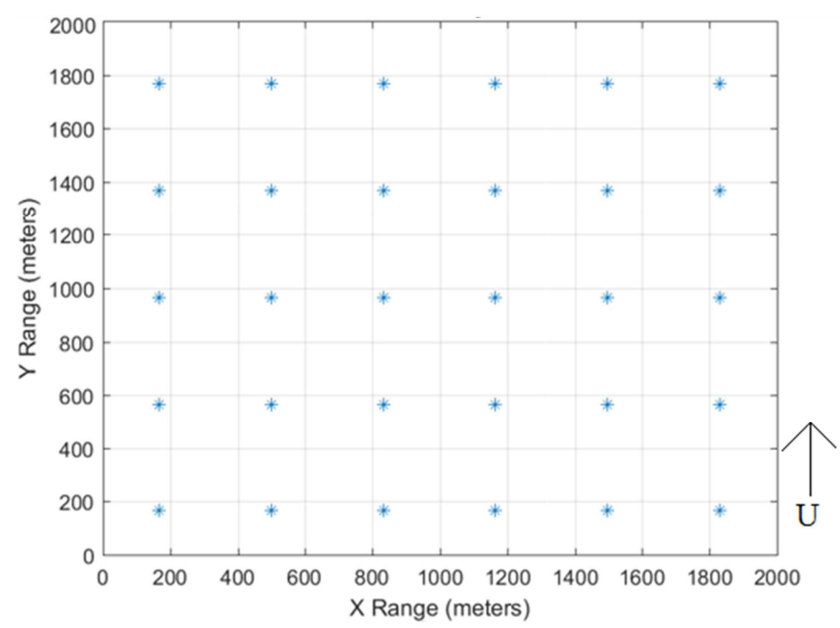

Figure 4. Customised turbine arrangement. 


\subsection{Customized Wind Turbine Layout Results}

Figure 4. presents the customized layout. The wind speed direction, $\mathrm{U}$, was taken to be in the $\mathrm{y}$-axis direction as indicated in figure 4. As stated earlier in chapter 2 , turbines are spaced with equal distances between each other. In the $\mathrm{x}$-axis direction; they are spaced with a separation distance of $333.3 \mathrm{~m}$ and with a separation distance of $400 \mathrm{~m}$ in the $\mathrm{y}$-axis direction.

In this arrangement, there are 6 columns with each having 5 turbines. Only 6 turbines from this arrangement receive freestream wind speed and the other 24 turbines are in the wake of the turbines placed before them. 5 turbines are placed in one line in the wind speed direction.

Power generated from the customized layout is 863.098 $\mathrm{kW}$. This power was obtained by manually entering turbines coordinates to the code for calculating the power and objective function value that is illustrated by the flowchart in figure 2 . When the wake effect is not considered, the maximum power that can be obtained from a wind farm of 30 turbines is 1500 $\mathrm{kW}$, obtained by multiplying the turbine rated power in table 2 by 30 . Comparing the power output from this layout to the maximum possible power, the equal spacing arrangement reduces the output power with $42 \%$.

\subsection{Genetic Algorithm Wind Turbine Layout Results}

Figure 5 illustrates the optimal arrangement of turbines produced by Genetic Algorithm (GA). The number of turbines placed to receive freestream wind speed is increased to 7 as compared to 6 in the customized layout in figure 4

A maximum number of turbines place in one line in the wind speed direction is 2 , which is less than the number of turbines placed in one line in the customized layout in which the number of turbines placed in one line in the wind speed direction is 5 .

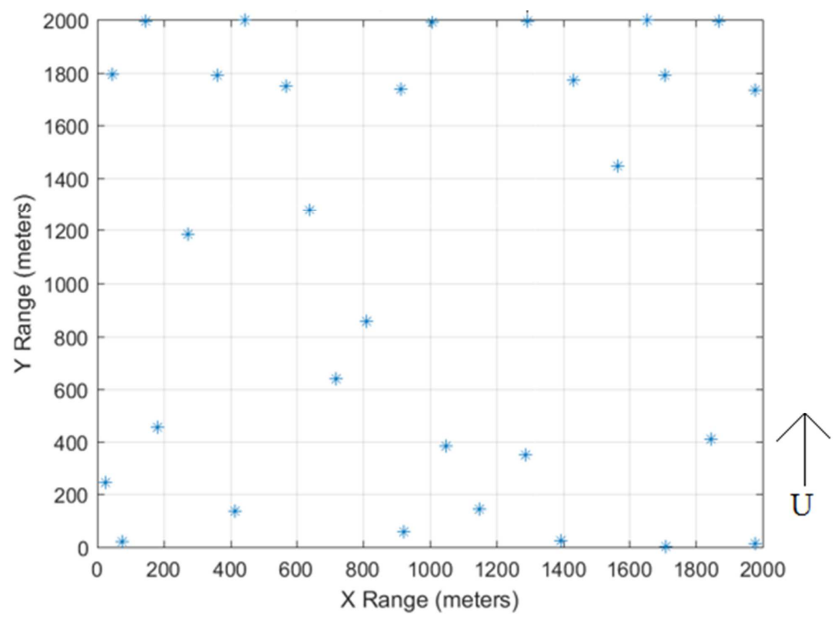

Figure 5. Genetic Algorithm turbine arrangement.

There is a large gap on the right side of the wind farm area between turbines at the bottom of the wind farm and turbines at the top of the wind farm. In the gap between the top and bottom turbines on the left side of the wind farm, there are two turbines. This explains why fewer turbines are placed on the top left corner area as compared to the turbines placed on the top right corner. The presence of the gap in the wind farm allows for wind speed to recover to a better value.

Power generated from the GA layout is $1296.286 \mathrm{~kW}$. Comparing the power output from the arrangement by GA to the maximum possible power, GA arrangement reduces the output power with $13.5 \%$.

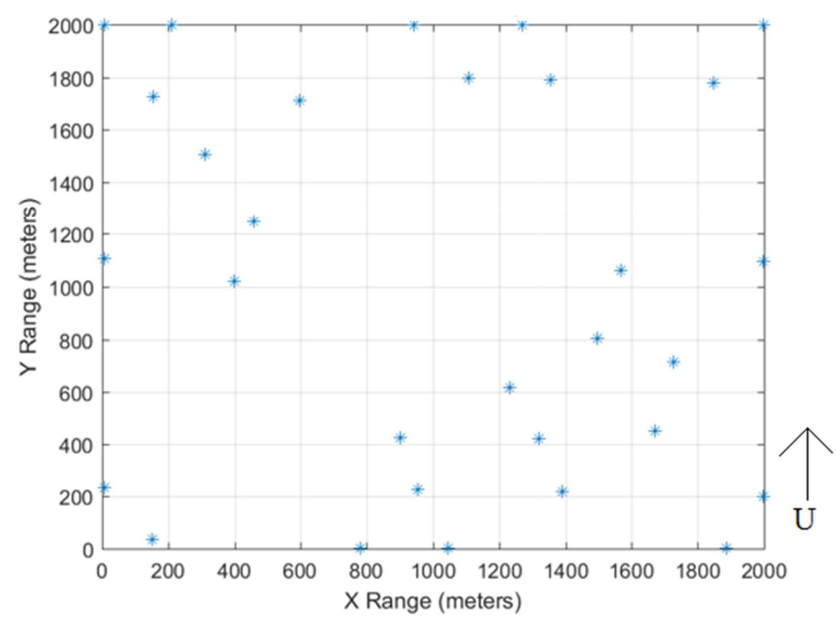

Figure 6. Particle Swarm Optimization turbine arrangement.

\subsection{Particle Swarm Optimization Turbine Layout}

Figure 6 is the optimal arrangement of turbine generated by Particle Swarm Optimization (PSO) algorithm. From this arrangement, 7 turbines are arranged in a manner that will allow them to receive freestream wind speed, this is like the arrangement generated by GA and at the same time, the number of turbines receiving freestream wind speed is greater than the number of turbines receiving freestream wind speed in the customized layout.

There is a group of turbines arranged at the bottom right corner area while only a few turbines are placed at the top right corner area. A few number of turbines are placed at the top right corner area because a large number of turbines placed before them, this is because the wake approaching the back of the wind farm is too much so placing a large number of turbines in this area, will lead to less power produced.

Many turbines are placed on the left top corner of the wind farm area while fewer turbines are placed at the bottom left corner. This is because the wake approaching the back of the wind farm does not have much negative effect on turbines placed at that area because wind passes through a few number of turbines placed before them on the bottom left corner area of the wind farm.

Power generated from PSO layout is $1300.668 \mathrm{~kW}$. Comparing the power output from the arrangement by PSO to the maximum possible power, PSO arrangement reduces the output power with $13.3 \%$.

\section{Conclusion}

In this paper Genetic Algorithm and Particle Swarm Optimization were used to generate the optimal arrangement of turbines in a wind farm. A customized wind farm layout 
with turbines arranged with equal spacing was developed and compared to the wind farm layout with turbines that were optimally arrange by using Genetic Algorithm and Particle Swarm Optimization algorithm.

Comparing the arrangement of the optimization algorithms to the arrangement with equal spacing between turbines, the optimization algorithms arrangement resulted in a more optimal arrangement. With GA optimal arrangement, power generated was increased by $50.2 \%$ while from PSO power generated was increased by $50.7 \%$.

Regarding wind farm power losses, the wind farm layout from optimization algorithms results in less power loss as compared to the power loss of the customized layout. The customized wind farm layout results in the most power losses of $43 \%$, GA wind farm layout followed with losses of $13.5 \%$ while PSO results in minimum losses as compared to other wind farm layouts with $13.3 \%$.

The results from GA and PSO slightly differ, this can be observed from the difference between the power generated from GA and PSO layout which is $4.4 \mathrm{~kW}$. With this little difference in powers, it can be confidently stated that the results are valid. Based on the results, it can be observed that optimization algorithms lead to better power yield when used turbines layout in a wind farm.

\section{Nomenclature}

$f(v)$
$k$
$\mathrm{c}$
$\left(x_{m}, y_{m}\right)$ and $\left(x_{n}, y_{n}\right)$
$\mathrm{N}$
$X_{\max }$ and $Y_{\max }$
$\mathrm{WPn}$
$\mathrm{C}$
$\mathrm{r}_{\mathrm{c}}$
$\mathrm{r}_{\mathrm{i}}$
$\mathrm{nL}$
$\mathrm{X}$
$\eta$
$\rho$
$\mathrm{A}$
$u$
$i$
$u_{i}$

Probability of wind speed

The shape parameter

The scale parameter in $\mathrm{m} / \mathrm{s}$

Cartesian coordinates of the $m t h$ and $n t h$ wind turbine

Number of variables

Maximum length of the area in the $x$ and $y$ directions

Net value of power production

Variable part of the total wind farm investment costs

Interest rate

Rate of inflation

Number of times interests of loans have to be paid per year

wind farm lifetime in years

Turbine efficiency

Air density

Swept area of the turbine rotor

Wind velocity

Specific turbine

Wind speed at specific turbine

\section{References}

[1] R. Mouton, "Namibia renewws commitment to renewable energy," Windhoek Observer, 28 July 2017. [Online]. Available:

http://www.observer.com.na/index.php/national/item/8479-na mibia-renews-commitment-to-renewable-energy. [Accessed 26 October 2017].

[2] P. N. Alireza Emami, "New Approach on Optimazation in Placement of Wind Turbines within Wind Farm by Genetic Algorithm," Renewable Energy, vol. 35, pp. 1559-1564, 2010.

[3] G. M. Masters, Renewable and Efficient Electric Power Systems, 2nd Edition, Hoboken, New Jersey: Weley-IEEE Press, 2013.

[4] A. C. R. Saini, "Statistical Analysis of Wind Speed Data Using Weibull Distribution Parameters," in 1st International Conference on Non Conventional Energy, Kalyani, 2014.

[5] C. P. a. B. D. G. Mosetti, "Optimization of wind turbine positioning in large windfarms by means of a genetic algorithm," Journal of Wind Engineering and Industrial Aerodynamics, vol. 51, no. 1, pp. 105 - 116, 1994.

[6] I. M. a. D. Borissova, "Wind turbines type and number choice

[7] H. D. S. a. J. C. Smith, "A polyhedral study of the generalized vertex packing problem," Mathematical Programming, vol. 107, no. 3, pp. 367 - 390, 2005.

[8] P. R. a. G. L. A. Tessauro, "State of the Art of Wind Farm Optimisation," DTU Wind Energy.

[9] J. W. a. X. Z. Chunqui Wan, "Optimal Micro-sitting of Wind Farms by Particle swarm Optimization," Renewable Energy, vol. 1, no. 47, pp. 198 - 205, 2010.

[10] P. F. G. C. L. T. B. J. L. a. H. A. M. Pierre-Elouan Réthoré, "TOPFARM: Multi-fidelity optimization of wind farms," John Wiley \& Sons, Ltd., Roskilde, 2013.

[11] Á. G. R. J. C. M. R. S. a. M. B. P. J. Serrano González, “A New Tool for Wind Farm Optimal Design," in IEEE Bucharest Power Tech Conference, Bucharest, 2009.

[12] C. A. P. M. Bryony L. Dupont, "Optimization Of Wind Farm Layout And Wind Turbine Geometry Using A Multilevel Extended Pattern Search Algorithm That Accounts For Variation In Wind Shear Profile Shape," In Proceedings of The Asme 2012 International Design Engineering Technical Conferences \& Computers And Information In Engineering Conference, Chicago, 2012. 
[13] M. H. a. M. A. S. A Grady, "Placement of Wind Turbines using Genetic Algorithms," Renewable Energy, vol. 30, no. 2, pp. 259 - 270, 2005.

[14] M. Y. H. A. R. N. R. a. M. N. M. N. Rabia Shakoor, "Wind Farm Layout Optimization by Using Definite Point Selection and Genetic Algorithm," in IEEE International Conference Power \& Energy (PECON), 2014.
[15] Jun Wang and Xing Zhang, "Wind farm micro-sitting by Gaussian particle swarm optimization with local search strategy," Renewable Energy, no. 48, pp. 276-, 2012.

[16] J. W. a. X. Zhang, "Optimal Micro-siting of Wind Farms by Particles Swarm Optimisation," in Lecture Notes in Computer Science, Beijing, 2010. 